\section{Exercise, dominant hand and neurodegeneration}

\author{
Satoshi Kuwabara, Sonoko Misawa
}

Some neurodegenerative diseases, such as amyotrophic lateral sclerosis (ALS) and Parkinson's disease (PD), characteristically present with asymmetrical symptoms, and previous studies have shown that there is concordance for side of onset and handedness. ${ }^{12}$ Separately, the markedly higher incidence of ALS has been reported in athletes, particularly in professional soccer players in Italy, and American football players in the USA. These observations have led to debate about the possible role of physical exercise in the pathogenesis of ALS, and direct effects mediated by excitotoxicity, oxidative stress and mitochondrial dysfunction. ${ }^{2} 3$ Further evidence to support the role of exercise or overuse is the split hand syndrome in ALS, whereby the thenar muscles are more preferentially affected than the hypothenar muscles, possibly because humans use the thenar muscles much more frequently. ${ }^{3}$

In a study involving 343 limb-onset ALS patients, for upper-limb onset patients the concordance rate for dominant hand and onset side was $64 \%(p<0.0006)$, whereas such concordance was not evident in lower-limb onset patients. ${ }^{1}$ A meta-analysis of 10 studies including 4405 PD patients has revealed that $59.5 \%$ of right-handed and $59.2 \%$ of left-handed patients had predominant PD symptoms in the dominant hand (OR 2.13). ${ }^{2}$ Whereas possible causal factors in these disorders may predominantly affect the central neural network, these results suggest that exercise influences the pathogenesis of neurodegeneration in ALS and PD.

Department of Neurology, Graduate School of Medicine, Chiba University Chiba, Japan

Correspondence to Dr Satoshi Kuwabara, Department of Neurology, Graduate School of Medicine, Chiba University, 1-8-1 Inohana, Chuo-ku, Chiba 260-8670, Japan; kuwabara-s@faculty.chiba-u.jp
In peripheral nerve or muscle disorders, overwork weakness has been proposed for muscular dystrophy as early as the 1970 s. $^{4}$ For peripheral neuropathies, CharcotMarie-Tooth (CMT) disease has been frequently investigated, and several previous studies involving 22-80 patients showed mixed results. Some studies using the MRC scores support dominant involvement of the dominant hand, but others using amplitudes of compound muscle action potentials did not show significant differences between the dominant and non-dominant hands. ${ }^{5}$

The study by Piscosquito et $a l^{5}$ investigated the hypothesis that dominant limbs may be predominantly affected in 271 patients with CMT1A, by post hoc analyses of large data in Italian-UK ascorbic acid trial for CMT1A. Although this trial failed to show the beneficial effects of ascorbic acid, the study was carefully designed; the dominant side for hand and foot was checked, and bilateral homonymous muscles were tested. The results showed no differences between the two sides in quantitative muscle strength measured in the first dorsal interosseous, abductor pollicis brevis, tibialis anterior and triceps surae, irrespective of age, disease duration and clinical disability. The authors conclude that their data do not support the overwork hypothesis in CMT1A patients and recommend active rehabilitation. CMT1A is a dysmyelinating neuropathy, and activity-dependent axonal hyperpolarisation may induce conduction block, and thereby muscle fatigue, but at least voluntary activity does not appear to enhance neurodegeneration.

The findings may at first glance not seem surprising because patients with CMT usually show symmetric polyneuropathy, but this study supports the view that exercise or physical activity in daily life would not significantly affect disease progression and axonal degeneration in CMT1A, and presumably in other types of CMT. Appropriate rehabilitation remains an effective treatment for CMT and related neuropathies. In contrast, the clear lateralisation of symptoms and association with handedness in ALS and PD remain part of a more profound mystery, and the vulnerability in the central neural circuit, particularly in the dominant cerebral hemisphere, must be further investigated.

Acknowledgements This work was supported in part by the Health and Labour Sciences Research Grant on Intractable Diseases (Neurodegenerative Diseases) from the Ministry of Health, Labour and Welfare of Japan.

Contributors SK wrote the first draft, and SM revised it.

Competing interests None.

Patient consent Obtained.

Provenance and peer review Commissioned; internally peer reviewed.

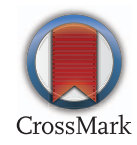

To cite Kuwabara S, Misawa S. J Neurol Neurosurg Psychiatry 2014;85:1291.

Received 24 February 2014

Revised 27 March 2014

Accepted 29 March 2014

Published Online First 25 April 2014

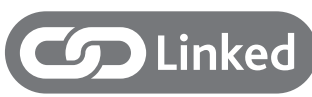

- http://dx.doi.org/10.1136/jnnp-2014-307598

I Neurol Neurosurg Psychiatry 2014;85:1291. doi:10.1136/jnnp-2014-307924

\section{REFERENCES}

1 Turner MR, Wicks P, Brownstein CA, et al. Concordance between site of onset and limb dominance in amyotrophic lateral sclerosis. J Neurol Neurosurg Psychiatry 2011;82:853-4.

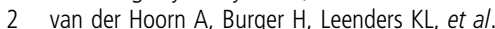
Handedness correlates with the dominant Parkinson side: a systematic review and meta-analysis. Mov Disord 2012;27:206-10.

3 Eisen A, Kuwabara S. The split hand syndrome in amyotrophic lateral sclerosis. I Neurol Neurosurg Psychiatry 2012;83:399-403.

4 Johnson EW, Braddom R. Over-work weakness in facioscapulohuumeral muscular dystrophy. Arch Phys Med Rehabil 1971;52:333-6.

5 Piscosquito G, Reilly NM, Schenone A, et al. Is overwork weakness relevant in Charcot-Marie-Tooth disease? J Neurol Neurosurg Psychiatry 2014;85:1343-7. 